Connaissez vous le livre: Plantes médicinales de l'lle de Rodrigues?

Non? C'est bien dommage!

Car dans l'archipel des Mascareignes, doté d'un immense lagon et d'un aéroport à Plaine Corail, l'île de Rodrigues est baignée par les eaux de l'Océan Indien et offre aux visiteurs une flore d'une grande richesse et des centaines de plantes médicinales. Les Rodriguais sont descendants d'une poignée de colons français, d'Indiens et d'esclaves africains venus se poser là quand la Compagnie des Indes Orientales colonisa l'île de France (aujourd'hui Maurice) et l'Île Bourbon (La Réunion).

Nous voici loin des festivités et de la remise des prix L'Oréal-Unesco, qui se sont déroulées le 22 février, place de Fontenoy à Paris en présence de Koïchiro Matsuura, Directeur général de l'Unesco, et de Sir Lindsay Owen-Jones, Président de L'Oréal. Au cours de cette soirée, animée par la journaliste Christine Ockrent, une conférence a été donnée par Suzan Greenfield, professeure de pharmacologie de I'Université d'Oxford et membre de la Chambre des Lords. En fait, nous n'en sommes pas si loin pourtant, car parmi les cinq femmes chercheures émérites venues des cinq continents et récompensées ce jour-là par le jury présidé par Pierre Gilles de Gennes pour leurs exceptionnels travaux, se trouvait une professeure de l'Île Maurice qui avait justement écrit ce livre et en a publié un second: Plantes médicinales et aromatiques des îles de l'Océan Indien [1].

\section{Ameenah Gurib-Fakim}

Ameenah Gurib-Fakim est en effet professeure de chimie organique, pro vice-chancelière de I'Université

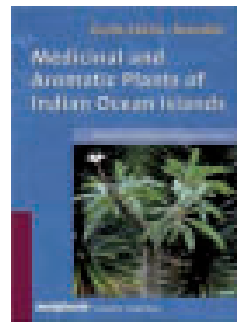

Médecine/Sciences,

9 , rue Basse,

54330 Clérey-sur-Brénon,

France.

de Maurice. Elle a consa-

sgilgenkrantz@ cré une bonne partie de sa medecinesciences.org vie à la flore de son pays natal, l'île Maurice.

Âgée de 48 ans, elle parle parfaitement le français et l'anglais, mais a également pris la défense du créole. Après ses études secondaires, elle a étudié la chimie à l'Université d'Exeter (Royaume-Uni), puis a travaillé dans une compagnie américaine, la Dow Chemical Company, avant de retourner à Maurice où elle a gravi tous les échelons de la carrière universitaire.

L'Île Maurice et ses voisines de l'Océan Indien (Madagascar, la Réunion et Rodrigues) possèdent des centaines de plantes ayant des propriétés médicinales. Le «baume de I'île Plate», par exemple, ou Psiadia terebinthina est un buisson aux feuilles collantes riches en flavonoïdes, des pigments antioxydants qui, non seulement accélèrent la cicatrisation des plaies, mais protègent les vaisseaux et ont des propriétés anti-asthmatiques. Les recensements qu'elle a effectués, ainsi que les analyses scientifiques des propriétés antibactériennes, antifongiques et antidiabétiques de diverses plantes ont permis d'ouvrir la voie à leur utilisation en médecine. Ameenah Gurib-Fakim intervient dans un projet de culture d'Aloe Vera avec la société Mon Désert-Mon trésor au sud de l'île Maurice, afin d'analyser les principes actifs de l'aloès et d'aider à la mise en place de la plantation et de la production. Elle travaille aussi sur la margose, ou melon amer (Momordica charantia), cette plante grimpante de la famille des Cucurbitacées qui aurait des propriétés anti-diabétiques - le diabète est, avec l'hypertension artérielle et les maladies infec- 
tieuses un des fléaux de l'île Maurice -, ainsi que sur le jaquier (Artocarpus heterophyllus) et la nêfle du Japon (Eriobotrya japonica). Son souhait est de développer la culture des plantes médicinales à Maurice L'originalité de ses recherches lui valut des débuts difficiles. Elle a dû se battre pour se faire reconnaître. Son activité - en médecine - rappelle un peu celle de Muhammad Yunus - en économie. Bien que ce Bangladais n'ait guère été pris au sérieux pendant des années, il finit par être nominé pour le prix Nobel d'économie (et reçut le prix Nobel de la paix en 2006) pour avoir créé la Grameen Bank, cette banque offrant des microcrédits aux exclus du système bancaire pour les pauvres (surtout des femmes). À l'instar de Muhammad Yunus, la persévérance et l'importance qu'attache Ameenah Gurib-Fakim aux phyto-médicaments justifie sa réussite. Elle est aussi très consciente des risques de biopiratage et souhaite que les grands groupes pharmaceutiques internationaux, capables d'isoler les principes actifs, acceptent d'en faire profiter gratuitement les habitants des îles d'où ces plantes sont originaires.

Les quatre autres lauréates n'ont sans doute pas moins de mérite, mais leur cursus, beaucoup plus classique les ont moins mises en valeur auprès des journalistes de la grande presse, surtout attirés par l'orientation très écologique et éthique de cette ravissante Mauricienne musulmane.

\section{Ligia Gargallo}

Ligia Gargallo (Chili, Amérique Latine), née en 1934, est récompensée pour ses études sur les solutions de molécules flexibles à longue chaîne. Professeure au département de chimie et physique à l'Université catholique pontificale du Chili, à Santiago, elle a étudié notamment des macromolécules et des polymères naturels et synthétiques. Ses études sur la conformation et le comportement thermodynamique des polymères, leur transformation à l'état visqueux ou élastique ont des applications dans des domaines tels que la technologie, la médecine et l'environnement.

\section{Mildred Dresselhaus}

Mildred Dresselhaus (États-Unis, Amérique du Nord) a grandi à New York. Diplomée de l'Université de Chicago, elle est ensuite entrée au MIT (Massachusetts Institute of Technology), Cambridge, Massachusetts, દ́tats-Unis. Ses travaux ont porté sur la physique des solides et en particulier la conceptualisation des nanotubes de carbone. Grâce à leur petite taille, leur solidité mécanique extrêmement élevée et une grande conductivité électrique, ceux-ci peuvent se substituer à divers matériaux (cristaux liquides dans les écrans plats) ou être utilisés comme éléments biocompatibles (électrodes ou transporteurs de matériaux biologiques). Millie Dresselhaus est aussi la première femme faite Docteur Honoris Causa de l'Université française Pierre et Marie-Curie.

\section{Margaret Brimble}

Margaret Brimble (Nouvelle-Zélande, Asie-Pacifique) est professeure à la chaire de chimie organique et médicinale de l'Université d'Auckland. Ses études ont porté sur de nombreux produits naturels complexes, comme les toxines trouvées chez les mollusques. Elle a consacré une grande partie de son travail à la synthèse des substances utiles à la conception de médicaments destinés à soigner Alzheimer, épilepsie, hypertension, accidents vasculaires et cancer.

\section{Tatiana Maksimovna Birshtein}

Tatiana Maksimovna Birshtein (Russie, Europe) est née à Léningrad en 1928. Elle est actuellement professeure à l'Institut des composés macromoléculaires de Saint-Pétersbourg et membre de l'Académie russe des Sciences. Elle a découvert des propriétés d'auto-organisation de divers systèmes de polymères utilisés dans la fabrication du nylon, du polyester, de la mousse de polystyrène, du Plexiglas ou du Téflon. T. Birshtein a aussi beaucoup participé à l'enseignement au département de physique.

\section{Le partenariat L'Oréal-Unesco}

Ces cinq lauréates 2007 ont été sélectionnées à l'issue des délibérations du Jury international du Prix 2007 L’Oréal-Unesco en sciences de la matière, et ont reçu 100000 dollars chacune.

«Le partenariat L'Oréal-Unesco est exemplaire par la vision de la science qu'il cherche à promouvoir pour garantir l'avenir de la planète », a déclaré le Directeur général de I'Unesco. « II est exemplaire par sa conception du rôle que des organisations comme les nôtres ont à jouer dans ce formidable défi ».

En effet, ces «Prix L’Oréal-Unesco pour les femmes et la science» pourraient servir de modèle à d'autres initiatives. Ils ont pour objectif de faire reconnaître la contribution de chercheures émérites au progrès scientifique et d'encourager les carrières féminines dans des sciences réputées «dures», longtemps considérées comme le domaine réservé des hommes. À ce jour, 47 femmes originaires de 21 pays ont été distinguées.

Au total, plus de 350 chercheures à travers le monde ont bénéficié de ces prix. Lancé par L'Oréal et l'Unesco en 1998, ce programme pionnier a donné lieu à de multiples projets destinés à soutenir les femmes de science, encourager les progrès de la connaissance et améliorer le développement des sociétés à l'échelle mondiale. En outre, 15 Bourses internationales L'Oréal-Unesco sont également attribuées chaque année à de jeunes post-doctorantes. Par ailleurs, depuis 2001, plus de 200 doctorantes ont bénéficié des Bourses nationales L'Oréal, auxquelles sont associées les Commissions nationales pour l'Unesco. De son côté, l'Oréal Canada (basé à Montréal) a offert deux bourses de 20000 \$ à Linda Furlini et Mariela 
